\title{
A Comprehensive Framework for the Computer-Aided Planning and Optimisation of Manufacturing Processes for Functional Graded Components
}

\author{
Marcus Petersen and Jürgen Gausemeier \\ Heinz Nixdorf Institute, Product Engineering, University of Paderborn, \\ Fuerstenallee 11, 33102 Paderborn, Germany \\ \{marcus.petersen, juergen.gausemeier\}@uni-paderborn. de
}

\begin{abstract}
In this contribution, we present a framework for the computer-aided planning and optimisation of manufacturing process chains for functional graded components. The framework is divided into three modules - the "Component Description", the "Expert System" for the synthetisation of several manufacturing process chains and the "Modelling and Process Chain Optimisation". The Component Description module enhances a standard computer-aided design (CAD) model by a voxel-based representation of the intended graded properties. The Expert System synthesises manufacturing process steps stored in the knowledge base to generate alternative process chains. All these process chains are capable of producing components according to the enhanced CAD model. They consist of a sequence of heating-, cooling-, and forming manufacturing processes. The interdependencies between the component and the applied manufacturing processes as well as between the processes themselves need to be considered. For that purpose the Expert System utilises an ontology. The ontology represents all the interdependencies in a structured way and connects the information of the knowledge base via relations. The third module performs the evaluation of the generated manufacturing process chains. To accomplish this, the parameters of each process step are optimised according to the component specification, whereby the result of the best parameterization is used as a representative value. Finally the process chain which is capable of producing a functional graded component in an optimal way regarding to the property distributions of the component description is presented by means of a dedicated specification technique.
\end{abstract}

Keywords: Manufacturing Process Planning, Functional Graded Components, Expert System, Specification Technique, Sustainable Production.

\section{Introduction}

Functional gradation denotes a continuous distribution of properties over at least one of the spatial dimensions of a component consisting of only one material. This distribution is tailored according to the intended application of the component [1]. 
Application areas for the use of functional graded components can be found for example in the automotive industry. Car interior door panels for instance are usually plastic materials that are supposed to absorb the impact energy of a lateral crash to an assured extent. The resulting deformation however must in no case lead to an injury of the car's passengers. To achieve a desired deformation behaviour it is necessary to assign exactly defined material properties to specific locations of the door panel. By a functional gradation, e.g. of the hardness, the functionality of the component can be considerably extended. The formerly purely decorative interior door panel becomes a functional element of the passive vehicle safety.

Functional graded components provide a resource-conserving alternative for modern composite materials [1] and therefore offer high potential to achieve a sustainable production. Instead of using post-processing steps to create the composites and their graded properties, the gradation is produced during their moulding process. This process integration for example shortens the manufacturing process chain for the production of the component and increases the energy efficiency significantly.

The production of functional graded components requires complex manufacturing process chains, such as thermo-mechanically coupled process steps [1]. While there are several material scientific approaches on how to develop an isolated process step to achieve a certain material structure, the holistic design of connected manufacturing process chains is much more difficult. For that purpose in section two an exemplary manufacturing process chain will be used to demonstrate our approach. To realise the full potentials of functional gradation, a computer-aided framework for the planning and optimisation of this manufacturing process chains will be introduced in the following subsection, whereupon the hierarchical process chain synthetisation as part of the Expert System will be presented in section three. Section four summarises the approach and identifies the significant future research challenges.

\section{Functional Graded Components}

\subsection{Exemplary Manufacturing Process Chain}

The manufacturing process chains for functional graded components are characterised by strong interdependencies between the components and the applied manufacturing processes as well as between the process steps themselves. According to the presented interior door panel (cf. section 1), a manufacturing process chain for self-reinforced polypropylene composites is used here as a demonstrator. This process chain uses a thermomechanical hot-compaction process to integrate the functional gradation into selfreinforced polypropylene composites by processing layered semi-finished textile products on a thermoplastic basis. The semi-finished textile products were previously stretched and provide a self-reinforcement based on a macromolecular orientation. This self-reinforcement leads to a sensitive behaviour regarding pressure and thermal treatments and is therefore essential for the functional gradation of the composite [1], [2].

Figure 1 shows the exemplary manufacturing process chain for self-reinforced polypropylene composites starting with a gradual preheating of the semi-finished textile products in a partially masked IR-preheating station. In the next step, the thermal gradation of 
the product will be enhanced due to consolidation by a special compression moulding tool. The tool was particularly design for thermo-mechanical gradation. For this reason, both tool halves can be tempered differentially and completely independent from each other. Furthermore this tool applies a mechanical gradation by a local pressure reduction of up to $30 \%$ due to the triangular geometry. A cooling phase is necessary before demoulding the self-reinforced polypropylene composite [3].

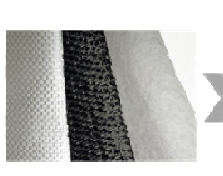

sernl-finished textile products
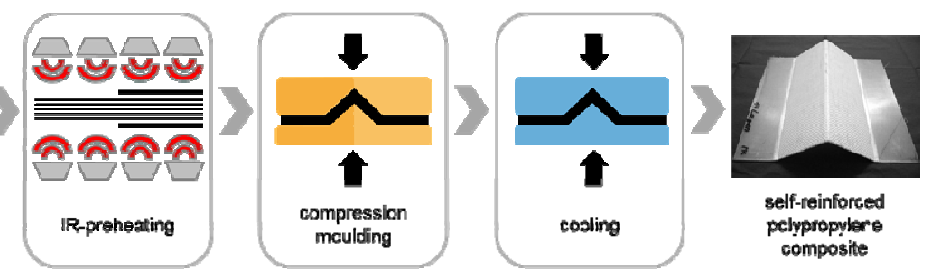

Fig. 1. Exemplary manufacturing process chain for self-reinforced polypropylene composites

\subsection{Comprehensive Planning Framework}

The exemplary manufacturing process chain for self-reinforced polypropylene composites is characterised by strong interdependencies (cf. section 2.1). These interdependencies are typical for the production of components with functionally graded properties and need to be considered. Therefore a comprehensive planning framework for the planning and optimisation of manufacturing process chains is under development. This framework integrates several methods, tools and knowledge obtained by laboratory experiments and industrial cooperation projects in which the concept of functional gradation has been analysed. The planning process within the framework is continuously assisted by the modules "Component Description“" "Expert System" and "Modelling and Process Chain Optimisation" [1].

Figure 2 gives an overview about the structure of the planning framework and the information exchanges between the modules. The input information for the manufacturing process planning is provided by the computer-aided design (CAD) model of the component and the intended graded properties. Based on this information, several alternative process chains for the manufacturing of the component are synthesised by means of the framework. After this, the process parameters of each process chain are optimised based on empirical models. The best manufacturing process chain is described using a dedicated specification technique for production systems in the last step of the planning process [4].

The Component Description module enables the desired graded properties to be integrated into the CAD model of the component. The model usually consists of geometric features (e.g. cylinder or disc), which will be extracted after loading the model. These features allow the framework to consider the geometry of the whole component and to pre-select reasonable gradients according to the geometry. This preselection increases the efficiency of describing the intended gradient since the manufacturing planner can directly provide the desired graded properties by modifying the 
parameters of the proposed gradients. If the CAD model does not contain any geometric feature or the user does not want to use one of the pre-selected gradients, the component is divided into small volume elements. These so called voxels enable the component model to be locally addressed and can be used as supporting points for the function-based integration of the component's graded properties [5].

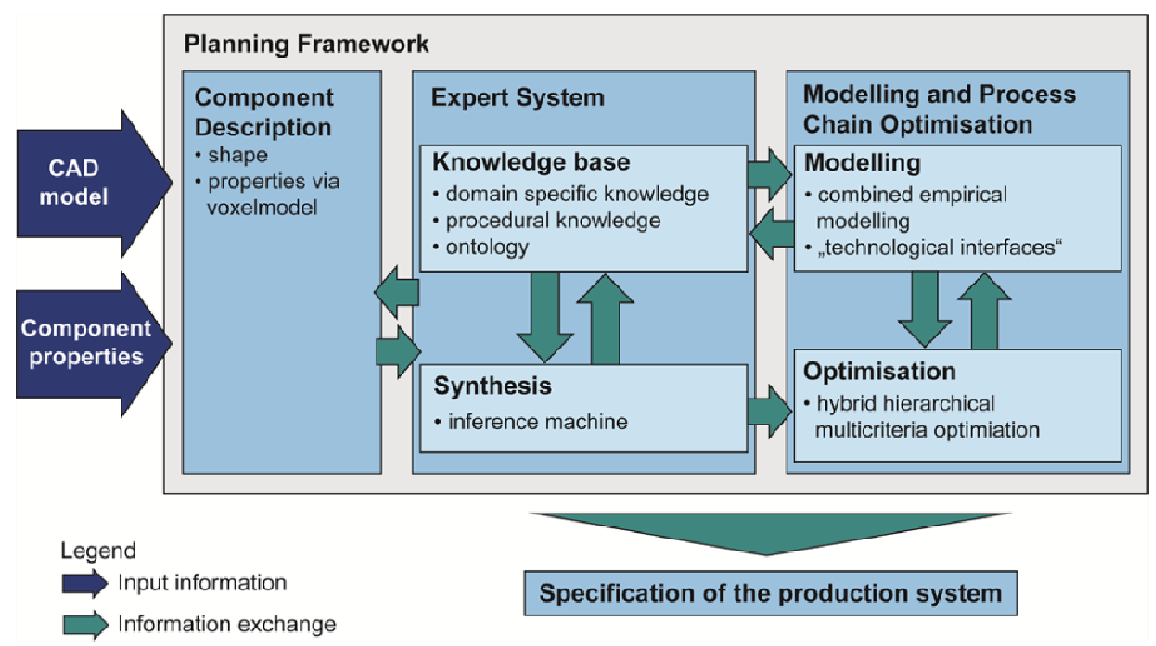

Fig. 2. Planning Framework for the computer-aided planning and optimisation of manufacturing processes for functional graded components

Based on the enhanced CAD model of the first module, the Expert System synthesises several alternative process chains for manufacturing the component. For that purpose all the manufacturing processes available in the knowledge base are filtered according to the component description such as material, geometry or the desired graded properties (e. g. hardness or ductility). To realise this filtering process, the content of the knowledge base is structured by an ontology. The ontology classifies the process steps with regard to their characteristics and connects the information of the knowledge base via relations between the content elements. An inference machine is applied to draw conclusions from the ontology, especially with respect to the varied interdependencies between the manufacturing processes. These conclusions provide the main information for connecting the several process steps of the knowledge base during the synthetisation of reasonable manufacturing process chains according to the enhanced CAD model of the component. The synthetisation of several alternative manufacturing process chains by a hierarchical process chain synthesis is described in section three.

The exemplary manufacturing process chain for self-reinforced polypropylene composites is for example characterised by the fact that the initial material temperature, which is adjusted during the IR-preheating process in preparation of the compression moulding process has a strong influence on the mouldability of the component. 
Those and all the other interdependencies mentioned above need to be considered during the pairwise evaluation of process steps to ensure the compatibility of the synthesised process chains for the manufacturing of the component. All process chains with incompatible process steps are disregarded. Thus the result of the Expert System module is a set of several alternative process chains which are capable of producing the component [1].

The parameters of a preferred set of manufacturing process chains are optimised by means of the Modelling and Process Chain Optimisation module [1]. To accomplish this, predictions of empirical models based on several experiments, measurements and simulations of samples provide a comprehensive solution space (cf. [6]). Modern empirical modelling techniques are then used as surrogates for the processes and a hybrid hierarchical multi-objective optimisation is utilised to identify the optimal setup for each process step of a manufacturing process chain. In the context of functional gradation, design and analysis of computer experiments (DACE) models have proven to show a very good prediction quality [7], [8].

After all, the process chain that is capable of producing a functional graded component in the best way regarding to the component description is described using a dedicated specification technique. This fundamental specification is based on a process sequence and a resource diagram [9].

Figure 3 shows an extract of the optimised process sequence for the manufacturing of self-reinforced functional graded polypropylene composites with an example set of process step parameters for the compression moulding. Further information about the specification technique can be found in [9].

The next section gives an overview about the underlying principles of the manufacturing process chain synthetisation within the Expert System.
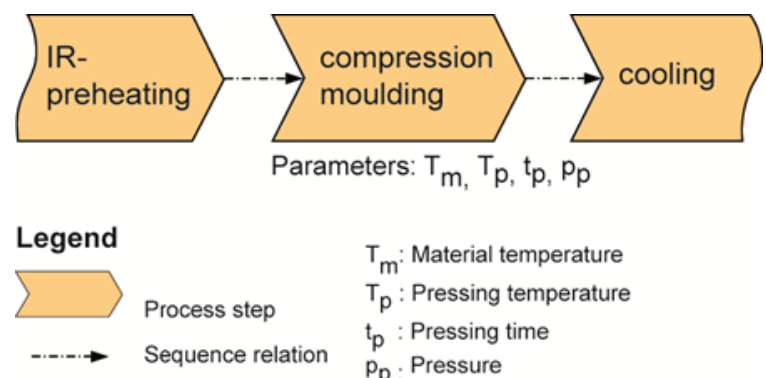

$T_{m}$ : Material temperature
$T_{p}:$ Pressing temperature
$t_{p}:$ Pressing time
$p_{p}$ : Pressure

Fig. 3. Part of the optimised process sequence for the manufacturing of self-reinforced polypropylene composites 


\section{Hierarchical Process Chain Synthetisation}

The Expert System within the planning framework synthesises several alternative manufacturing process chains for functional graded components in a hierarchical way. This synthetisation is assisted by two steps - the "Core Process Selection" and the "Process Chain Synthetisation". The component requirements provided by the Component Description module, such as the enhanced CAD model, the material or general requirements (e.g. the surface quality) constitute the product attributes for the requirements profile of the component. This radar chart profile [10] and also the component requirements represent the input information for the Expert System (cf. Figure 4).

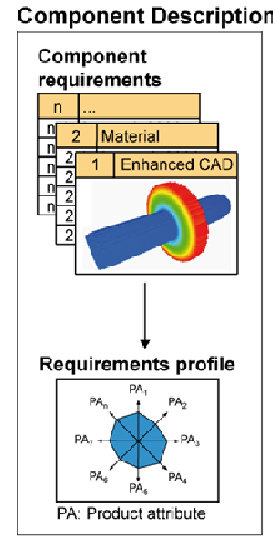

Modelling and Process Chain Optimisation

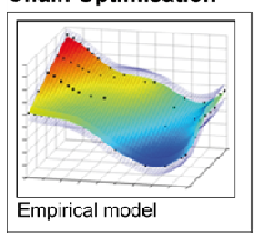

\section{Expert System: Core Process Selection and Process Chain Synthetisation}
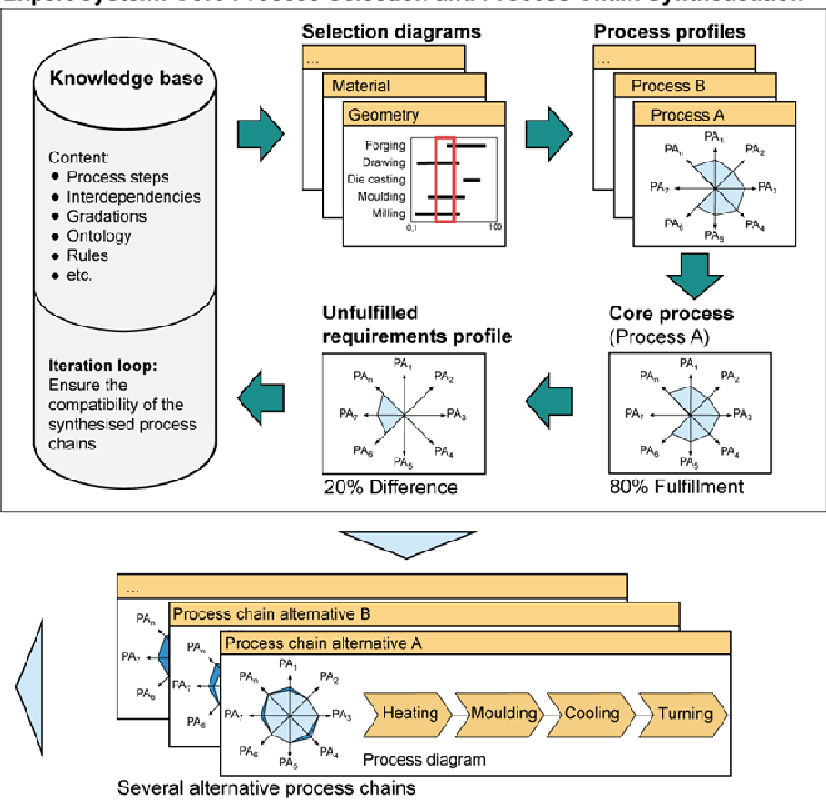

Fig. 4. Hierarchical process chain synthetisation as part of the Expert System module

\subsection{Core Process Selection}

The Core Process Selection (according to [11]) marks the first synthetisation loop of the Expert System and results in the core process for the manufacturing of the component. Thereby the process step which fulfils the requirements of the component according to the requirements profile in the best way is selected to be the core process. This manufacturing process also establishes the root process i.e. the starting point for the hierarchic process chain synthetisation within the iteration loops of the Expert System (cf. section 3.2).

At first all the manufacturing process steps available in the knowledge base are structured according to each product attribute of the requirement profile for the manufacturing of the component. For this purpose the Expert System utilises matrix tables, 
in which the manufacturing processes are displayed in the rows and their ability range of the current product attribute is represented in the columns. These so called selection diagrams provide the basis for the automatic selection of the core process. Figure 5 shows an example of such a matrix table for the product attribute "tolerance".

Based on these selection diagrams, all the manufacturing processes which do not match the product requirements in the defined range are removed. For the other process steps, a process profile is created in addition to the requirement profile. The process profile is presented to the user within the planning framework to explain the results of the selection process. These profiles show the fulfilment of the component requirements by the given manufacturing processes in a comprehensive way (cf. Figure 4).

The manufacturing process step with the highest fulfilment of the product attributes is selected to be the core process and the unfulfilled requirements form the main input for the Process Chain Synthetisation as new requirement profile.

\begin{tabular}{|l|l|l|l|l|}
\hline \multirow{2}{*}{ Manufacturing Process } & \multicolumn{3}{|c|}{ Tolerance (mm) } \\
\hline Sand Casting & 0.01 & & \\
\hline Die Casting & & & & \\
\hline Investment Casting & & & & \\
\hline Forging & & & & \\
\hline $\begin{array}{l}\text { Extrusion } \\
\text { Sheet metal forming }\end{array}$ & & & & \\
\hline Powder metallurgy & & & & \\
\hline Electrical discharge machining & & & & \\
\hline Conventional machining & & & & \\
\hline Injection moulding & & & & \\
\hline Blow moulding & & & & \\
\hline Compression moulding & & & & \\
\hline Deep Drawing & & & & \\
\hline Polymer casting & & & & \\
\hline $\begin{array}{l}\text { Resin-transfer moulding } \\
\text { Filament winding }\end{array}$ & & & & \\
\hline Lamination techniques & & & \\
\hline Vacuum bag moulding & & & & \\
\hline
\end{tabular}

Fig. 5. Example of a selection diagram (according to [11]) 


\subsection{Process Chain Synthetisation}

The Process Chain Synthetisation starts only if the Core Process Selection ends up with some unfulfilled requirements. This step of the Expert System tries to reduce the unfulfilled requirements down to a minimum by creating several alternative process chains.

To create the process chains, the Expert System restarts the Core Process Selection as a loop, in which the unfulfilled requirements of a completed iteration loop provide the input information for the next iteration. This loop continues until no further process step can be found to fulfil the open requirements of the requirements profile.

After every iteration loop, a pairwise evaluation with the new selected manufacturing process and the already connected process steps is performed to ensure the compatibility of the synthesised process chain. If there is only one incompatible process step in the process chain a new alternative process chain will be started without this step, but with an own unfulfilled requirement profile. This new process chain will also be considered during the following iterations, whereby new selected process steps will be integrated in every suitable process chain. If the Expert System has to consider two or more alternative process chains, the Process Chain Synthetisation continues until no further process step can be found to fill up one of the open unfulfilled requirement profiles of the process chains.

The result of the hierarchical process chain synthetisation is a set of several alternative process chains which are all able to achieve the desired component requirements (cf. Figure 6).

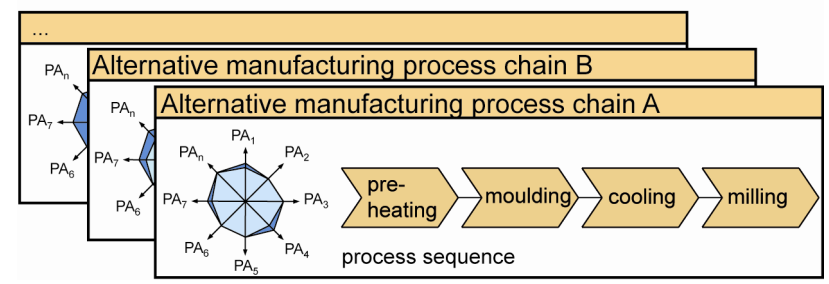

Fig. 6. Set of alternative process chains for the interior door panel given by the Expert System

\section{Conclusions and Outlook}

Functional graded components offer an innovative and sustainable approach for customisable smart products. Thus a comprehensive framework for the computer-aided planning and model-based optimisation of components with functional graded properties has been presented and demonstrated with an application example.

Future work includes the enhancement of the knowledge base with additional manufacturing process steps, materials and interdependencies as well as the adjustment of the ontology. Furthermore the inference rules of the expert system have to be 
expanded to realise the synthetisation of more complex manufacturing process chains and their pairwise evaluation. The Expert System of the comprehensive planning framework is able to automatically synthesise process chains for the manufacturing of a component with functionally graded properties. However the final selection of the best process chain for the specific production objective must still be conducted manually since it is not always obvious which alternative fulfils all the requirements according to the objective in the best way. The Analytic Hierarchy Process (cf. [12]) may offer an effective approach to handle the highly diverse characteristics of the decision criteria while not overstraining the decision process with data acquisition and examination.

Acknowledgement. The work in this contribution is based upon investigations of the Collaborative Transregional Research Centre (CRC) Transregio 30, which is kindly supported by the German Research Foundation (DFG).

\section{References}

1. Biermann, D., Gausemeier, J., Heim, H.-P., Hess, S., Petersen, M., Ries, A., Wagner, T.: Computer-Aided Planning and Optimisation of Manufacturing Processes for Functional Graded Components. In: Proceedings of the 1st International Conference on ThermoMechanically Graded Materials, Kassel, Germany, October 29-30 (2012)

2. Bledzki, A.K., Ries, A., Paßmann, D.: Functional graded self-reinforced polypropylene sheets. Polimery 56 (2011)

3. Paßmann, D.: Prozessinduzierte Gradierung eigenverstärkter Polypropylen-Faserverbunde beim Heißkompaktieren und Umformen, Dissertation, University of Kassel (2010)

4. Gausemeier, J., Bauer, F., Dettmer, D., Reyes Perez, M.: Planning of Manufacturing Processes for Graded Components. In: Proc. International Conference Product Property Prediction - P3, Dortmund, Germany, April 12-13 (2010)

5. Bauer, F., Dettmer, D., Gausemeier, J.: Feature-based component description for functional graded parts. In: 4th International Conference on Changeable, Agile, Reconfigurable and Virtual Production (CARV 2011), Montreal, Canada, October 02-05 (2011)

6. Wagner, T., Paßmann, D., Weinert, K., Biermann, D., Bledzki, A.K.: Efficient modeling and optimisation of the property gradation of self-reinforced polypropylene sheets within a thermo-mechanical compaction process. In: Proceedings of the 6th CIRP International Conference on Intelligent Computation in Manufacturing Engineering (ICME 2008), Naples, Italy, July 23-25 (2008)

7. Sieben, B., Wagner, T., Biermann, D.: Empirical Modeling of Hard Turning of AISI 6150 Steel Using Design and Analysis of Computer Experiments. Production Engineering - Research and Development 4, 115 (2010)

8. Wagner, T., Bröcker, C., Saba, N., Biermann, D., Matzenmiller, A., Steinhoff, K.: Analysis of a Thermomechanically Coupled Forming Process Using Enhanced Design and Analysis of Computer Experiments. In: Digital Proceedings of the International Conference on Design and Analysis of Computer Experiments (ENBIS EMSE), St-Etienne, France, July 01-03 (2009) 
9. Gausemeier, J., Dumitrescu, R., Kahl, S., Nordsiek, D.: Integrative Development of Product and Production System for Mechatronic Products. Robotics and Computer-Integrated Manufacturing 27(4) (2011)

10. Fallböhmer, M.: Generieren alternativer Technologieketten in frühen Phasen der Produktentwicklung. Dissertation, RWTH Aachen University, Shaker Verlag (2000)

11. Ashby, M.F.: Materials Selection in Mechanical Design - Das Original mit Übersetzungshilfen. Elsevier, Munich (2007)

12. Saaty, T.L.: The analytic hierarchy process - planning, priority setting, resource allocation. McGraw-Hill, New York (1980) 